Bull. Austral. Math. Soc.

VoL. $71(2005) \quad$ [517-526]

\title{
GENERATING FUNCTIONS RELATED TO THE OKAMOTO POLYNOMIALS FOR THE PAINLEVÉ IV EQUATION
}

\author{
HiROMICHI Goto and KenJI KaJIWARA
}

\begin{abstract}
We construct generating functions for the entries of Hankel determinant formula for the Okamoto polynomials which characterise a class of rational solutions to the Painlevé IV equation. Generating functions are characterised as asymptotic expansions of $\log$ derivative of $\mathrm{Ai}$ and $\mathrm{Bi}$, which are solutions of the Airy equation.
\end{abstract}

\section{INTRODUCTION}

In the theory of integrable systems, the structure of exact solutions are considered to reflect essential mathematical structures behind the equations. In the soliton theory, as clarified by the Sato theory [11], the dependent variables which are introduced intuitively in Hirota's method ( $\tau$ functions) are regarded as the most fundamental object, and determinant or Pfaffian structure of the $\tau$ functions is a reflection of the solution space and the transformation group acting on it. For the Painleve equations, it is considered that the $\tau$ functions and their determinant structure are of the same importance as the soliton equations, although the Painlevé equations are ordinary differential equations while the soliton equations are partial differential equations. In the series of works $[14,15,16,17]$, Okamoto has introduced the $\tau$ functions through the Hamiltonians, and studied their properties. He has shown that the $\tau$ functions satisfy the Toda equation in general, and that some classes of transcendental classical solutions (hypergeometric solutions, or special function solutions) can be expressible in terms of determinants whose entries are given in terms of special functions of hypergeometric type. Moreover, for algebraic (or rational) solutions, which are another class of classical solutions to the Painlevé equations, it has been revealed that the special polynomials that characterise the solutions are specialisations of the Schur functions or their generalisation (universal characters) by studying the determinant formula of Jacobi-Trudi type $[4,6,7,9,10,13]$.

For example, the Painlevé II equation $\left(\mathrm{P}_{\mathrm{II}}\right)$

$$
\frac{d^{2} u}{d x^{2}}=2 u^{3}-4 x u+4\left(\alpha+\frac{1}{2}\right)
$$

Received 10th March, 2005

The authors would like to thank Prof. Nalini Joshi and Dr. Marta Mazzocco for discussion and encouragement. This work is partially supported by the JSPS Grant-in-Aid for Scientific Research.

Copyright Clearance Centre, Inc. Serial-fee code: 0004-9727/05 \$A2.00+0.00. 
admits a one-parameter family of transcendental classical solution expressible in terms of the solution of the Airy equation when $\alpha$ is an integer, and rational solutions when $\alpha$ is a half-integer. As for the rational solutions, the following fact is known: let $T_{N}(N \in \mathbb{Z})$ be the polynomials which are generated by the Toda equation

$$
T_{N}^{\prime \prime} T_{N}-\left(T_{N}^{\prime}\right)^{2}=T_{N+1} T_{N-1}-x T_{N}^{2}, \quad T_{0}=1, \quad T_{1}=x, \quad '=\frac{d}{d x} .
$$

Then we have $T_{N}=T_{-N-1}$, and it is shown that the function

$$
u=\frac{d}{d x} \log \frac{T_{N+1}}{T_{N}},
$$

satisfy $\mathrm{P}_{\mathrm{II}}$ (1) with $\alpha=N+(1 / 2)$. The polynomials $T_{N}$ are called the Yablonski-Vorob'ev polynomials. It is known that the Yablonski-Vorob'ev polynomials admit the following determinant formula of Jacobi-Trudi type [6]:

(4)

$$
T_{N}=c_{N}\left|\begin{array}{cccc}
p_{N} & p_{N+1} & \cdots & p_{2 N-1} \\
p_{N-2} & p_{N-1} & \cdots & p_{2 N-3} \\
\vdots & \vdots & \ddots & \vdots \\
p_{-N+1} & p_{-N+2} & \cdots & p_{1}
\end{array}\right|, \quad \sum_{k=0}^{\infty} p_{k} \lambda^{k}=\exp \left[x \lambda+\frac{\lambda^{3}}{3}\right], \quad p_{n}=0(n<0)
$$

$$
c_{N}=(2 N-1)^{!}=(2 N-1) ! !(2 N-3) ! ! \cdots 3 ! ! .
$$

Since the Schur function $S_{Y}\left(t_{1}, t_{2}, t_{3}, \ldots\right)$ associated with the 2-core Young diagram $Y=(N, N-1, \cdots, 1)$ is given by the determinant in (4) with $p_{k}$ replaced by

$$
\sum_{k=0}^{\infty} p_{k} \lambda^{k}=\exp \sum_{n=1}^{\infty} t_{n} \lambda^{n}
$$

([11]), the above formula immediately implies that $T_{N}$ are nothing but the specialisation of 2-core Schur functions. Furthermore, since the 2-core Schur functions give rational solutions to Korteweg-de Vries or modified Korteweg-de Vries equation ([11]), this coincides with the fact that $P_{I I}$ can be derived from similarity reduction of the modified Korteweg-de Vries equation (see, for example, [1]). In this manner, the determinant formula is useful to clarify the essential property of the object.

On the other hand, it is known that $T_{N}$ admits the following Hankel determinant formula [6]:

$$
T_{N}=\left|\begin{array}{cccc}
a_{0} & a_{1} & \cdots & a_{N-1} \\
a_{1} & a_{2} & \cdots & a_{N} \\
\vdots & \vdots & \ddots & \vdots \\
a_{N-1} & a_{N} & \cdots & a_{2 N-2}
\end{array}\right|, \quad a_{0}=x, \quad a_{n}=a_{n-1}^{\prime}+\sum_{k=0}^{n-2} a_{k} a_{n-2-k} .
$$


If a determinant formula reflects some mathematical structure, what does this formula imply? In particular, what are the entries $a_{n}$ ? In order to investigate this problem, a generating function for $a_{n}$ was considered in [2], and the following result was obtained.

THEOREM 1.1. ([2]) We define the generating function $F(x, t)$ of $a_{n}(n \geqslant 0)$ by

$$
F(x, t)=\sum_{n=0}^{\infty} a_{n}(-2 t)^{-n} .
$$

Then, the following asymptotic expansion around $t \rightarrow \infty$ holds for any subsector of $|\arg t|<\pi / 2$ :

$$
F(x, t) \sim \frac{\partial}{\partial t} \log \theta(x, t), \quad \theta(x, t)=\mathrm{e}^{\left(2 t^{3}\right) / 3} \mathrm{Ai}\left(t^{2}-x\right),
$$

where $\mathrm{Ai}$ is the Airy function.

This result is quite suggestive, since log derivative of the Airy function is another particular solution to $P_{\mathrm{II}}$. In fact,

$$
u=\frac{d}{d x} \log \operatorname{Ai}\left(2^{1 / 3} x\right),
$$

satisfies $\mathrm{P}_{\mathrm{II}}$ (1) with $\alpha=0$. Is this phenomenon caused by an accident? In order to answer this question, it may be an important problem to investigate whether similar phenomena take place for other Painlevé equations or not.

In this article, we consider the Painlevé IV equation ( $\left.\mathrm{P}_{\mathrm{IV}}\right)$

$$
\frac{d^{2} y}{d x^{2}}=\frac{1}{2 y}\left(\frac{d y}{d x}\right)^{2}+\frac{3}{2} y^{3}+6 x y+\frac{9}{2}\left(x^{2}-\frac{4}{3} \alpha\right) y-\frac{\beta}{2 y},
$$

where $\alpha$ and $\beta$ are parameters, and the Okamoto polynomials which characterise a class of rational solutions for $P_{I V}$. We construct the Hankel determinant formula for the Okamoto polynomials and discuss the generating functions for the entries of determinants.

\section{OKAMOTO POLYNOMIALS AND HANKEL DETERMINANT FORMULA}

Let $Q_{n}(n \in \mathbb{Z})$ be polynomials generated by the following Toda equation.

$$
\begin{aligned}
Q_{n}^{\prime \prime} Q_{n}-\left(Q_{n}^{\prime}\right)^{2} & =Q_{n+1} Q_{n-1}-\left(x^{2}+2 n+1\right) Q_{n}^{2}, \\
Q_{0} & =1, \quad Q_{1}=x^{2}+1 .
\end{aligned}
$$

Then, it is known that $[\mathbf{1 6}, \mathbf{1 2}]$

$$
y=\frac{d}{d x} \log \frac{Q_{n+1}}{Q_{n}}-x,
$$

satisfies $\mathrm{P}_{\mathrm{IV}}$ (10) with $\alpha=n+1, \beta=1$. The polynomials $Q_{n}$ are called the Okamoto polynomials. A Jacobi-Trudi type determinant formula for $Q_{n}$ is discussed in $[7,13,12]$, and it is shown that $Q_{n}$ are specialisation of 3-core Schur functions.

Let us construct the Hankel determinant formula for $Q_{n}$. A Hankel determinant formula for the general solution of the Toda equation has been presented in [5] as follows. 
THEOREM 2.1. ([5].) Let $\left\{\tau_{n}\right\}_{n \in Z}$ functions satisfying the Toda equation:

$$
\tau_{n}^{\prime \prime} \tau_{n}-\tau_{n}^{\prime 2}=\tau_{n+1} \tau_{n-1}-\psi \varphi \tau_{n}^{2}, \quad \tau_{-1}=\psi, \quad \tau_{0}=1, \quad \tau_{1}=\varphi .
$$

Let $\left\{a_{n}\right\}_{n \in \mathbb{N}},\left\{b_{n}\right\}_{n \in \mathbb{N}}$ be sequences defined by the recursion relation

$$
a_{n}=a_{n-1}^{\prime}+\psi \sum_{k=0}^{n-2} a_{k} a_{n-k}, \quad b_{n}=b_{n-1}^{\prime}+\varphi \sum_{k=0}^{n-k} b_{k} b_{n-k}, \quad a_{0}=\varphi, \quad b_{0}=\psi
$$

respectively. Then $\tau_{n}$ is expressed as

$$
\tau_{n}= \begin{cases}\operatorname{det}\left(a_{i+j-2}\right)_{i, j \leqslant n} & n>0 \\ 1 & n=0 \\ \operatorname{det}\left(b_{i+j-2}\right)_{i, j \leqslant|n|} & n<0\end{cases}
$$

Applying Theorem 2.1 to the Toda equation for $Q_{n}$ (11), we have the following formula.

\section{PROPOSITION 2.2.}

$$
\begin{aligned}
Q_{n} & = \begin{cases}\operatorname{det}\left(a_{i+j-2}\right)_{1 \leqslant i, j \leqslant n}, & n \geqslant 1 \\
1 & n=0,-1 \\
\operatorname{det}\left(b_{i+j-2}\right)_{1 \leqslant i, j \leqslant|n|-1}, & n \leqslant-1\end{cases} \\
a_{n} & =a_{n-1}^{\prime}+2 x a_{n-1}+\sum_{k=0}^{n-2} a_{k} a_{n-2-k}, \quad a_{0}=x^{2}+1, \quad a_{1}=2 x^{3}+4 x, \\
b_{n} & =b_{n-1}^{\prime}-2 x b_{n-1}+\sum_{k=0}^{n-2} b_{k} b_{n-2-k}, \quad b_{0}=x^{2}-1, \quad b_{1}=2 x^{3}-4 x
\end{aligned}
$$

Proof: For technical reason, we separate the cases $n>0$ and $n<0$. Putting $Q_{n}=\mathrm{e}^{-n x^{2}} R_{n}$ in (11), we have

$$
\begin{aligned}
R_{n}^{\prime \prime} R_{n}-\left(R_{n}^{\prime}\right)^{2} & =R_{n+1} R_{n-1}-\left(x^{2}+1\right) R_{n}^{2}, \\
R_{-1} & =\mathrm{e}^{-x^{2}}, \quad R_{0}=1, R_{1}=\mathrm{e}^{x^{2}}\left(x^{2}+1\right) .
\end{aligned}
$$

Applying Theorem 2.1 to (20) with $n>0$, we have

$$
R_{n}=\operatorname{det}\left(\tilde{a}_{i+j-2}\right), \quad \tilde{a}_{n}=\tilde{a}_{n-1}^{\prime}+\mathrm{e}^{-x^{2}} \sum_{k=0}^{n-2} \tilde{a}_{k} \tilde{a}_{n-k}, \quad \tilde{a}_{0}=\mathrm{e}^{x^{2}}\left(x^{2}+1\right) .
$$

Now putting $\tilde{a}_{n}=\mathrm{e}^{x^{2}} a_{n}$ and noticing that $R_{n}=\mathrm{e}^{n x^{2}} Q_{n}$, we obtain the formula for $n>0$ as

$$
Q_{n}=\operatorname{det}\left(a_{i+j-2}\right), \quad a_{n}=a_{n-1}^{\prime}+2 x a_{n-1}+\sum_{k=0}^{n-2} a_{k} a_{n-k}, \quad a_{0}=x^{2}+1
$$


For the case of $n<0$, putting $Q_{n}=\mathrm{e}^{-(n+1) x^{2}} S_{n+1}$, we have

$$
\begin{aligned}
S_{n}^{\prime \prime} S_{n}-\left(S_{n}^{\prime}\right)^{2} & =S_{n+1} S_{n-1}-\left(x^{2}-1\right) S_{n}^{2}, \\
S_{-1} & =\mathrm{e}^{-x^{2}}\left(x^{2}-1\right), \quad S_{0}=1, \quad S_{1}=\mathrm{e}^{x^{2}} .
\end{aligned}
$$

Applying Theorem 2.1 to (23) with $n<0$, we get the formula for $n<0$ by similar calculations.

\section{Generating functions}

3.1. MAIN RESULT. Let $I_{\nu}(z)$ be the modified Bessel function

$$
I_{\nu}(z)=\sum_{n=0}^{\infty} \frac{(z / 2)^{\nu+2 k}}{\Gamma(k+1) \Gamma(k+\nu+1)}, \quad|z|<\infty, \quad|\arg z|<\pi .
$$

We define the functions $\operatorname{Ai}(z)$ and $\operatorname{Bi}(z)$ by

$$
\begin{aligned}
& \operatorname{Ai}(z)=\frac{z^{1 / 2}}{3}\left[I_{-1 / 3}\left(\frac{2 z^{3 / 2}}{3}\right)-I_{1 / 3}\left(\frac{2 z^{3 / 2}}{3}\right)\right], \\
& \operatorname{Bi}(z)=\frac{z^{1 / 2}}{3}\left[I_{-1 / 3}\left(\frac{2 z^{3 / 2}}{3}\right)+I_{1 / 3}\left(\frac{2 z^{3 / 2}}{3}\right)\right],
\end{aligned}
$$

respectively. It is known that $\mathrm{Ai}(z)$ and $\mathrm{Bi}(z)$ are independent solutions of the Airy equation

$$
\frac{d^{2} u}{d z^{2}}=z u
$$

and that they admit the following asymptotic expansion around $z \sim \infty([8])$ :

$$
\begin{aligned}
& \operatorname{Ai}(z) \sim U_{-}(z)|\arg z|<\pi \\
& \operatorname{Bi}(z) \sim U_{+}(z) \quad|\arg z|<\frac{\pi}{3}
\end{aligned}
$$

where

$$
U_{ \pm}(z)=\frac{1}{2 \sqrt{\pi}} \exp \left( \pm \frac{2 z^{3 / 2}}{3}\right) z^{-1 / 4} \sum_{n=0}^{\infty} \frac{(1 / 6)_{n}(5 / 6)_{n}}{n !}\left( \pm\left(4 z^{3 / 2}\right) / 3\right)^{-n}
$$

Now we define the generating functions for the entries $a_{n}$ and $b_{n}$ of the Hankel determinant formula of $Q_{n}$ obtained in Proposition 2.2 by

$$
F(x, t)=\sum_{n=0}^{\infty} a_{n} t^{-3 n}, \quad G(x, t)=\sum_{n=0}^{\infty} b_{n} t^{-3 n} .
$$

Then the main result of this article is stated as follows. 
THEOREM 3.1. Define the functions $\theta_{1}(x, t), \theta_{2}(x, t)$ by

$$
\left\{\begin{array}{l}
\theta_{1}(x, t)=\operatorname{Bi}\left(\frac{t^{4}}{4}-x t\right) \exp \left(-\frac{t^{6}}{12}+\frac{x t^{3}}{2}\right), \\
\theta_{2}(x, t)=\operatorname{Ai}\left(\frac{t^{4}}{4}+x t\right) \exp \left(\frac{t^{6}}{12}+\frac{x t^{3}}{2}\right),
\end{array}\right.
$$

respectively. Then, the following asymptotic expansions hold around $t \sim \infty$ :

$$
\begin{array}{ll}
F(x, t) \sim \frac{t^{4}}{x-t^{3}}\left[\frac{\partial}{\partial t} \log \theta_{1}(x, t)-\frac{x^{2}}{t}\right], & |\arg t|<\frac{\pi}{12}, \\
G(x, t) \sim \frac{t^{4}}{x+t^{3}}\left[\frac{\partial}{\partial t} \log \theta_{2}(x, t)+\frac{x^{2}}{t}\right], & |\arg t|<\frac{\pi}{4} .
\end{array}
$$

3.2. Riccati equation. In the following we give the proof of Theorem 3.1. The strategy for proof is described as follows:

(1) We derive Riccati equations for $F$ and $G$ from the recursion relations (18) and (19), respectively.

(2) We then linearise the Riccati equations by a standard technique to yield the Airy equation.

(3) Finally we identify the functions by investigating asymptotic behaviours.

Proposition 3.2. $F(x, t)$ and $G(x, t)$ satisfy the folloiwng Riccati equations,

$$
\begin{aligned}
& t^{3} \frac{\partial F}{\partial x}=-F^{2}+t^{3}\left(t^{3}-2 x\right) F-t^{6}\left(x^{2}+1\right), \\
& t^{3} \frac{\partial G}{\partial x}=-G^{2}+t^{3}\left(t^{3}+2 x\right) G-t^{6}\left(x^{2}-1\right),
\end{aligned}
$$

respectively.

Proof: (34) can be derived as follows:

$$
\begin{aligned}
F^{2} & =\sum_{n=0}^{\infty}\left(\sum_{k=0}^{\infty} a_{k} a_{n-k}\right) t^{-3 n}=\sum_{n=0}^{\infty}\left(a_{n+2}-a_{n+1}^{\prime}-2 x a_{n+1}\right) t^{-3 n} \\
& =\left(t^{6} F-t^{6} a_{0}-t^{3} a_{1}\right)-\left(t^{3} \frac{\partial F}{\partial x}-t^{3} a_{0}^{\prime}\right)-2 x\left(t^{3} F-t^{3} a_{0}\right) \\
& =-t^{3} \frac{\partial F}{\partial x}+t^{3}\left(t^{3}-2 x\right) F-t^{6}\left(x^{2}+1\right),
\end{aligned}
$$

where we have used (18) to derive the second equality. We obtain (35) by the similar calculation.

Since $F(x, t)$ and $G(x, t)$ are defined as formal series around $t \sim \infty$, it is useful to derive differential equations with respect to $t$. For this purpose we present the following lemma. 
LEMMA 3.3. (1) $a_{n}$ and $b_{n}$ satisfy the following recursion relations,

$$
\begin{aligned}
& a_{n}^{\prime}=x a_{n-1}^{\prime}+(3 n+1) a_{n-1} \\
& b_{n}^{\prime}=-x b_{n-1}^{\prime}-(3 n+1) b_{n-1}
\end{aligned}
$$

respectively.

(2) $F(x, t)$ and $G(x, t)$ satisfy the following linear differential equations,

$$
\begin{aligned}
& \left(x-t^{3}\right) \frac{\partial F}{\partial x}=t \frac{\partial F}{\partial t}-4 F-2 x t^{3} \\
& \left(x+t^{3}\right) \frac{\partial G}{\partial x}=t \frac{\partial G}{\partial t}-4 G+2 x t^{3}
\end{aligned}
$$

respectively.

The first statement can be proved by a simple induction. The second statement follows immediately from (36) and (37).

From Lemma 3.3, we obtain the Riccati equations with respect to $t$.

PROPOSITION 3.4. $F(x, t)$ and $G(x, t)$ satisfy the following Riccati equations

$$
\begin{aligned}
& t \frac{\partial F}{\partial t}=-\frac{x-t^{3}}{t^{3}} F^{2}+\left(-t^{6}+3 t^{3} x-2 x^{2}+4\right) F+t^{3}\left(t^{3} x^{2}+t^{3}-x^{3}+x\right) \\
& t \frac{\partial G}{\partial t}=-\frac{x+t^{3}}{t^{3}} G^{2}+\left(t^{6}+3 x t^{3}+2 x^{2}+4\right) G+t^{3}\left(-t^{3} x^{2}+t^{3}-x^{3}-x\right)
\end{aligned}
$$

respectively.

3.3. LINEARISATION AND IDENTIFICATION OF FUNCTIONS. We obtain the Airy equation by linearisation of the Riccati equations by standard technique.

\section{Proposition 3.5. (1) Putting}

$$
F=t^{3} \frac{v_{x}}{v}-x t^{3}+\frac{t^{6}}{2}
$$

the Riccati equation (34) reduces to the following linear equation.

$$
\frac{\partial^{2} v}{\partial x^{2}}=t^{3}\left(-x+\frac{t^{3}}{4}\right) v
$$

(2) Similarly, putting

$$
F=\frac{t^{4}}{x-t^{3}} \frac{v_{t}}{v}-x t^{3}+\frac{t^{6}}{2}
$$

the Riccati equation (40) reduces to the following linear equation.

$$
\frac{\partial^{2} v}{\partial t^{2}}=-\frac{3 t^{2}}{x-t^{3}} \frac{\partial v}{\partial t}+t\left(x-t^{3}\right)^{2}\left(-x+\frac{t^{3}}{4}\right) v
$$


(3) (43) and (45) are transformed into the Airy equation

$$
\frac{d^{2} v}{d z^{2}}=z v
$$

by the change of variable

$$
z=t\left(-x+\frac{t^{3}}{4}\right)
$$

A similar proposition holds for $G(x, t)$.

Proposition 3.6. (1) Putting

$$
G=t^{3} \frac{w_{x}}{w}+x t^{3}+\frac{t^{6}}{2}
$$

the Riccati equation (35) reduces to the following linear equation:

$$
\frac{\partial^{2} w}{\partial x^{2}}=t^{3}\left(x+\frac{t^{3}}{4}\right) w
$$

(2) Similarly, putting

$$
G=\frac{t^{4}}{x+t^{3}} \frac{w_{t}}{w}+x t^{3}+\frac{t^{6}}{2}
$$

the Riccati equation (41) reduces to the following linear equation:

$$
\frac{\partial^{2} w}{\partial t^{2}}=\frac{3 t^{2}}{x+t^{3}} \frac{\partial w}{\partial t}+t\left(x+t^{3}\right)^{2}\left(x+\frac{t^{3}}{4}\right) w
$$

(3) (49) and (51) are transformed into the Airy equation

$$
\frac{d^{2} w}{d z^{2}}=z w
$$

by the change of variable,

$$
z=t\left(x+\frac{t^{3}}{4}\right)
$$

We omit the proof since it is done by elementary calculations.

REMARK 3.7. Assertions (1) and (2) in Proposition 3.5 are consistent. Namely, (1) and (2) can be transformed to each other under the assumption that $(x, t)$ dependence of $u$ is given by $u=u(z)$. A similar remark also holds for Proposition 3.6.

Let us finally identify the function by considering its asymptotic behaviour around $t \sim \infty$. Solving (44) in terms of $v$, we have from (30)

$$
v(x, t)=\text { const. } \times \exp \left[\frac{1}{12} t^{6}-\frac{1}{2} x t^{3}\right] \times \frac{1}{t} \times \exp \left[-\sum_{n=0}^{\infty} \frac{x a_{n-1}-a_{n}}{3 n} t^{-3 n}\right] .
$$


The function $v$ is given as linear combination of $\operatorname{Ai}(z)$ and $\operatorname{Bi}(z)$. We compare the above expression with (28) and (29). Noticing that we have from (47)

$$
\frac{2}{3} z^{3 / 2} \stackrel{\bullet \rightarrow \infty}{\sim} \frac{t^{6}}{12}-\frac{1}{2} x t^{3}+\cdots, \quad z^{-1 / 4} \stackrel{\bullet}{\sim} \frac{\sqrt{2}}{t}\left(1+\frac{x}{t^{3}}+\cdots\right),
$$

we find that $u$ should be

$$
u(x, t) \sim U_{+}(z),
$$

by comparing the exponential factors. This proves the statement regarding $F$ in Theorem 3.1. The statement for $G$ can be proved in a similar manner by using Proposition 3.6 .

\section{CONCLUding REMARKS}

In this article, we have shown that the generating functions associated with the Hankel determinant formula for Okamoto polynomials can be characterised by the log derivative of solutions of the Airy equation. It is an interesting observation that $\mathrm{Bi}$ and $\mathrm{Ai}$, the solutions of Airy equation with different asymptotic behaviours, appear in opposite directions with respect to $n$.

We have posed the question as to whether the appearance of the log derivative of the Airy function in the generating function associated with Yablonski-Vorob'ev polynomials is an accident or not. The result of this article appears to imply that the answer is "No". It may be an interesting problem to characterise generating functions associated with special polynomials for other Painleve equations in terms of the log derivative of special functions of hypergeometric type.

If this phenomena is not caused by accident, we should clarify the reason and mechanism underlying it. Unfortunately we cannot give an answer to this problem yet. Recently, Hankel determinant formula for generic solutions to $\mathrm{P}_{\mathrm{II}}$ and the generating functions of its entries have been discussed in [3]. It is shown that the generating functions are also characterised by log derivative of solutions of certain linear differential equations, which are nothing but the auxiliary linear problem for $P_{I I}$. This result is not a conclusive answer to the problem, but it may imply some (unknown) correspondence between the Painlevé equations and their auxiliary linear problems. At least, it may be true that there is a nontrivial mathematical structure behind Hankel determinant formulae, which deserves further study.

\section{REFERENCES}

[1] M.J. Ablowitz and H. Segur, Solitons and the inverse scattering transform, SIAM Studies in Applied Mathematics 4 (SIAM, Philadelphia, 1981). 
[2] K. Iwasaki, K. Kajiwara and T. Nakamura, 'Generating function associated with the rational solutions of the Painlevé II equation', J. Phys. A 35 (2002 L207-L211).

[3] N. Joshi, K. Kajiwara and M. Mazzocco, 'Generating function associated with the determinant formula for solutions of the Painlevé II equation', Astérisque (to appear). arXiv:nlin.SI/0406035.

[4] K. Kajiwara and T. Masuda, 'On the Umemura polynomials for the Painlevé III equation', Phys. Lett. A 260 (1999), 462-467.

[5] K. Kajiwara, T. Masuda, M. Noumi, Y. Ohta and Y. Yamada, 'Determinant formulas for the Toda and discrete Toda equations', Funkcial. Ekvac. 44 (2001), 291-307.

[6] K. Kajiwara and Y. Ohta, 'Determinant structure of rational solutions for the Painlevé II equation', J. Math. Phys. 37 (1996), 4162-4174.

[7] K. Kajiwara and $Y$. Ohta, 'Determinant structure of the rational solutions for the Painlevé IV equation', J. Phys. A 31 (1998), 2431-2446.

[8] N.N. Lebedev, Special functions and their applications (Prentice-Hall, Englewood Cliffs, 1965).

[9] T. Masuda, 'On a class of algebraic solutions to the Painlevé VI equation, its determinant formula and coalescence cascade', Funkcial. Ekvac. 46 (2003), 121-171.

[10] T. Masuda, Y. Ohta and K. Kajiwara, 'A determinant formula for a class of rational solutions of Painlevé V equation', Nagoya Math. J. 168 (2002), 1-25.

[11] T. Miwa, M. Jimbo and E. Date, Solitons. Differential equations, symmetries and infinite-dimensional algebras, Cambridge Tracts in Mathematics 135 (Cambridge University Press, Cambridge, 2000).

[12] M. Noumi, Painlevé equations through symmetry, Translations of Mathematical Monographs 223 (American Mathematical Society, Providence, 2004).

[13] M. Noumi and Y. Yamada, 'Symmetries in the fourth Painlevé equation and Okamoto polynomials', Nagoya Math. J. 153 (1999), 53-86.

[14] K. Okamoto, 'Studies on the Painlevé equations. I. Sixth Painlevé equation PI', Ann. Mat. Pura Appl. 146 (1987), 337-381.

[15] K. Okamoto, 'Studies on the Painlevé equations. II. Fifth Painlevé equation $P_{V}$ ', Japan. J. Math. 13 (1987), 47-76.

[16] K. Okamoto, 'Studies on the Painlevé equations. III. Second and fourth Painlevé equations, $P_{I I}$ and $P_{I V}$ ', Math. Ann. 275 (1986), 221-255.

[17] K. Okamoto, 'Studies on the Painlevé equations. IV. Third Painlevé equation $P_{1 I I}$ ', Funkcial. Ekvac. 30 (1987), 305-332.

Graduate School of Mathematics

Kyushu University

6-10-1 Hakozaki

Fukuoka 812-8581

Japan
Graduate School of Mathematics

Kyushu University

6-10-1 Hakozaki

Fukuoka 812-8581

Japan

and

School of Mathematics and Statistics F07

The University of Sydney

Sydney 2006

Australia 